\title{
CONCEITO DE ENFERMAGEM TRANSCULTURAL: ANÁLISE DE SEU DESENVOLVIMENTO EM UMA DISSERTAÇÃO DE MESTRADO
}

\author{
THE CONCEPT OF TRANSCULTURAL NURSING: THE ANALYSIS OF ITS \\ DEVELOPMENT IN A MASTER'S DEGREE DISSERTATION \\ EL CONCEPTO DE ENFERMERIAA TRANSCULTURAL : ANÁLISIS DE SU \\ DESARROLLO EN UNA DISERTACIÓN DE MAESTRÍA
}

Maria Veraci Oliveira Queiroz

Lorita Marlena Freitag Pagliuca²

\begin{abstract}
Resumo: Estudo bibliográfico-analitico, objetivando analisar o conceito de enfermagem da Teoria de Leininger e o desenvolvimento conceitual de Enfermagem presente na dissertação de mestrado escolhida para o referido estudo. Utilizamos o modelo de análise e avaliação de teorias de Barnum (1998), em relação à clareza e consistência do conceito, associado a questionamentos sobre a Enfermagem. O conjunto de enunciados, presentes no conceito de Enfermagem Transcultural de Leininger e na dissertação, mostra o foco da enfermagem e a relação intrinseca com o cuidado, o qual deve ser desenvolvido coerente com a cultura. Demonstra, ainda, que a Enfermagem tem um corpo de conhecimentos especificos à profissão que orienta o cuidado ao cliente, valorizando a individualidade, a subjetividade e a visão de mundo. Concluimos que o estudo proporcionou validar conceitos abstraidos da Teoria da Universalidade e Diversidade Cultural do cuidado e sua utilização na pesquisa, conforme os critérios analisados.
\end{abstract}

PALAVRAS CHAVE: conceito de enfermagem, cultura, cuidar/cuidado

\section{INTRODUÇÃO}

A enfermagem como uma profissão em desenvolvimento está comprometida na formação de uma base de conhecimentos que dê apoio à sua prática. As teorias compõem parte desse conhecimento que orienta a prática e articula o pensar e o fazer da profissão, promovendo-a como uma ciência em continuo crescimento. Esta articulação é defendida por Barnum (1998), ao explicar que o conhecimento de enfermagem surge da prática, ambos devem formular teorias e estas devem direcionar a prática. Portanto, teoria, conhecimento e prática devem estar interrelacionadas, dando suporte à profissão.

As teorias de enfermagem podem ser entendidas como tentativas de solucionar problemas relacionados ao processo saúde-doença e como possibilidades de entender o homem em seu meio-ambiente (interno e externo). A enfermagem atua neste contexto como co-participante do cuidado humano. Nas idéias de Barnum (1998), uma teoria é uma construção, que organiza alguns fenômenos, descreve ou explica a enfermagem. É um caminho para caracterizar e apontar os componentes essenciais que dão identidade aos fenômenos da profissão.

Os conceitos são os elementos usados para produzir teorias. Na afirmação de (HICKMAN, 2000 , p. 11), "Os conceitos são palavras que representam a realidade e facilitam a nossa capacidade de comunicação sobre ela". A autora classifica como conceitos empíricos quando

\footnotetext{
1 Professora do Curso de Enfermagem da Universidade Estadual-Ceará. Doutoranda em Enfermagem na UFC

2 Professora Titular do Departamento de Enfermagem e da Pós Graduação da Universidade Federal do Ceará
} 
estes são observados ou percebidos pelos sentidos; e abstratos, quando não são observáveis, ou seja, não podem ser vistos ou tocados. Neste caso, temos a oportunidade de mentalizar o objeto referido pelo conceito.

No desenvolvimento de teorias de enfermagem, há quatro conceitos básicos, que incluem homem/indivíduo, sociedade/ambiente, saúde e enfermagem. Estes conceitos formam o metaparadigma de enfermagem, representados abstratamente como conteúdo essencial que influencia e determina a sua prática. (HICKMAN, 2000).

Nas idéias das autoras ora referenciadas, a teoria em si é formada por conceitos que envolvem valores, experiências, conhecimentos, propósitos e um contexto social. Estes atributos ,organizados sistematicamente, representam o modelo teórico, no qual a enfermagem desempenha suas ações.

$\mathrm{Na}$ oportunidade de estudar os critérios para análise de teorias e modelos de enfermagem, na disciplina Análise Critica das Teorias de Enfermagem, em função dos estudos feitos, despertamos para a análise do conceito de enfermagem presente na Teoria da Universalidade $\mathrm{e}$ Diversidade Cultural do Cuidado (TUDCC), por entender que esse sistema suscita valiosa contribuição ao trazer reflexões e delinear orientações para a prática. Com efeito, esboçamos o seguinte objetivo: analisar o conceito de Enfermagem da Teoria de Leininger e o desenvolvimento conceitual de Enfermagem Transcultural, presentes no texto-base da dissertação de mestrado escolhida para o presente ensaio.

\section{COMPREENSÃO IDEOLÓGICA DOS AUTORES}

A construção do conhecimento reúne vários elementos distintos. Para obtermos um conteúdo sistematizado e compreensivo de um objeto em estudo, precisamos de multi-referências associadas a partir de uma realidade empirica e abstrata, que contêm a subjetividade e a intersubjetividade dos autores referenciados. Neste estudo, incluimos o pensamento de Bárbara Barnum (1998), M. Leininger (1991) e V. Queiroz (1998).

Teorias são construidas para explicar ou compreender um fenômeno, porém nenhuma teoria tem intenção de abarcá-lo em todas as suas dimensões. Nenhuma ciência dá conta de explicar ou compreender todo o fenômeno, porquanto o estudo de determinado objeto é delimitado em seu contexto no tempo e no espaço.

Em relação às teorias de enfermagem, Barnum (1998) faz as seguintes explicações: na enfermagem as teorias não descrevem a prática, mas idealizam um mundo no qual as ações são desenvolvidas da melhor maneira possivel. Uma teoria de enfermagem completa é a que possui contexto, conteúdo e processo. A autora destaca os quatros componentes que devem estar presentes nas teorias de enfermagem: a pessoa - que pode ser definida pelo paciente, grupo, sociedade ou sistema de cuidado à saúde; a enfermagem - como ciência e arte - é identificada pelas ações presentes no fenômeno que aponta os componentes essenciais que dão identidade a esta profissão; a saúde - deve estar determinado o real significado, denotando a versão de saúde como um continuum saúde-doença, déficit de saúde ou limitação da autocapacidade e, ainda, as dimensões existentes e se estão contempladas no relato da saúde; o ambiente - corresponde ao universo onde ocorre o fenômeno.

Barnum (1998) faz considerações gerais sobre as teorias e suas abordagens filosóficas, destacando como foco de atenção, o homem e suas dimensões de saúde em seu meio ambiente. Sendo o enfermeiro interventor da prática, tanto ele desenvolve como aplica as teorias. Para esta autora a análise de teorias de enfermagem visa a aprender, a interpretar, compreender a teoria, evitando memorizá-la. Para nortear a avaliação de teorias, a autora orienta alguns passos significativos, que reúnem vários elementos distintos, a saber: os critérios de avaliação perpassam pelas correntes de teste pessoal ou pela utilização de critérios relacionados à crítica externa e interna. Esta lida com os componentes de clareza, consistência, adequação, desenvolvimento lógico e nivel do desenvolvimento da teoria. 
Estratégias adicionais utilizadas para avaliação de teorias seguem os seguintes passos: identificar o maior elemento da teoria; determinar se a teoria é explicativa ou explanatória; determinar se a teoria descreve a enfermagem como ela é ou como deveria ser; se a teoria descreve o que a enfermeira faz ou o que deveria fazer; analisar se o foco de enfermagem paira sobre o conteúdo do conhecimento de enfermagem, nos métodos de desenvolvimento das ações, no conceito, no contexto e nas metas de enfermagem; identificar como o homem é abordado na teoria, se como paciente, ação de enfermagem ou a relação enfermeira-paciente. A autora ainda sugere avaliar se a teórica dá alguma definição inconsistente com a teoria. Estas questões são significativas para a análise e avaliação de teorias em si, e ainda, para elucidar sua aplicaçăo no desenvolvimento de pesquisa cientifica.

A Teoria da Universalidade e Diversidade Cultural do Cuidado foi desenvolvida por Leininger, pioneira da enfermagem transcultural. Desde a década de 50 , vem estudando como utilizar este conhecimento na prática de enfermagem. Com base nas disciplinas de Sociologia, Psicologia e Antropologia, construiu a Teoria da Universalidade e Diversidade Cultural do Cuidado. A teoria, também chamada de Transcultural, tem como proposta principal proporcionar o cuidado de acordo com a cultura, numa abordagem holistica, focalizando a enfermagem cientifica e humanistica. O cuidado cultural busca perceber a diversidade e universalidade do cuidado humano em relação à visão de mundo, estrutura social e outras dimensões e, então, descobrir o modo de prover cuidados culturalmente congruentes para diferentes pessoas, familia ou grupo cultural (LEININGER, 1978, 1991).

A possibilidade de estudar o cuidado na perspectiva transcultural foi possivel e necessária pelo desenvolvimento do método de pesquisa com estas caracteristicas - a etnoenfermagem. Centraliza o estudo nas pessoas ou clientes, relacionando ao cuidado e sua influência na saúde e bem-estar em diferentes culturas no mundo. Tem como objetivos descobrir, documentar, preservar e atentamente interpretar os significados e experiências dos diferentes grupos culturais (LEININGER, 1991).

Na teoria, encontramos o modelo transcultural Sunrise, Leininger (1991) o qual mostra como é desenvolvido o cuidado cultural congruente por meio de ações e decisões que envolvem: preservação / negociação do cuidado cultural; acomodação / negociação do cuidado cultural; repadronização / restruturação do cultural.

Neste modelo, a autora faz uma descrição dos componentes da teoria, mostrando como estes influenciam o estado de saúde e o atendimento prestado aos clientes de uma determinada cultura. Defende o uso desse modelo na orientação de pesquisas qualitativas com abordagem etnográfica, na perspectiva de explorar e descobrir a essência e o significado do cuidado (HICKMAN, 2000).

Queiroz (1998), em dissertação de mestrado, descreve as experiências da mãe e familiares no cuidado à saúde da criança e reflete sobre a situação da mãe-acompanhante acerca da necessidade de um programa de educação permanente de apoio às familias de crianças hospitalizadas. Teve como informantes os acompanhantes e outros familiares da unidade de pediatria de um hospital da rede pública do SUS, em Fortaleza-Ceará-Brasil, no periodo de janeiro a dezembro de 1997. É uma pesquisa baseada nos pressupostos da etnoenfermagem, inserta na Teoria da Universalidade e Diversidade Cultural do Cuidado, de Madeleine Leininger. A coleta e análise de dados foram fundamentadas no modelo de Observação - Participação - Reflexão (O-P-R). Os dados foram submetidos ao critério de organização e análise, tendo emergido dois temas culturais: Só a mãe conhece o filho - que destaca o papel da mãe no cuidado da criança, o seu reconhecimento de que exclusivamente ela conhece e entende $o$ filho, e por isso, sacrifica-se em alguns momentos para estar com ele. No cuidado diário, as mães mostram a relação de cuidados permanentes para mantê-lo saudável. O segundo tema O que passei foi pra mim ensinar - mostra que as diversas situações vivenciadas pela mãe serviram para que ela aprendesse a agir em situaçōes de saúde e doença do filho, tornando-a mais experiente e capaz de ajudá-lo no processo de ser saudável. Ficou claro que, nesse 
convivio intercultural, as mães aprendem condutas e práticas advindas de um saber popular e profissional. Mesmo havendo esta troca de experiências, poucas vezes os profissionais realizam intencionalmente e sem imposições as suas orientações.

\section{PERCURSO METODOLÓGICO}

Trata-se de um estudo bibliográfico-analitico, tendo como referenciais a Teoria de Enfermagem Transcultural, de Leninger (1991), a dissertação de mestrado de Queiroz (1998) e o modelo de análise e avaliação de teorias proposto por Barnum (1998). Utilizamos os critérios de clareza e consistência do conceito, associados a questionamentos sobre a Enfermagem.

Análise é definida por Melleis (1997) como a identificação de partes e componentes, os quais devem ser examinados em relação ao número de critérios identificados. Analisar tem o sentido de acompanhar o processo, compreendê-lo, apreendê-lo mais globalmente num moto continuo, criativo, na dinâmica intersubjetiva de penetração na sua intimidade, buscando os múltiplos significados com a possibilidade de negação de si mesmo (BURNHAM, citado por SOUZA 1998). Enfim, análise permite uma compreensão sobre o fenômeno investigado).

O modelo de análise de teorias reúne diversos aspectos e deve seguir uma organização sistemática, orientada por estudiosos do assunto. Barnum (1998), em sua proposta, explica que a análise perpassa pelas correntes de teste pessoal ou pela utilização de critérios relacionados à critica externa e interna. Em relação à crítica interna, orienta a utilização dos critérios de clareza, consistência, adequação, desenvolvimento lógico e nivel do desenvolvimento da teoria, consoante referidos linhas atrás. Optamos por esse critério, escolhendo os componentes de clareza e consistência. O significado de clareza, para a autora, está em quando o objeto analisado se apresenta de modo fácil para o entendimento do leitor, capaz de denotar todos os objetos a que o termo se refere e a consistência envolve a fundamentação da expressão e de seu emprego de único modo.

Complementando a análise, utilizamos estratégias adicionais sugeridas também por Bamum (1998), que se destinam a avaliar teorias baseadas em questionamentos. Assim, fizemos adaptações para a análise de conceitos, utilizando interrogações que se dispõem a: determinar se o conceito descreve a enfermagem como é ela ou como deveria ser; identificar se o conceito descreve o que a enfermeira faz ou o que deveria fazer, e analisar se o foco de enfermagem paira sobre o conteúdo do conhecimento de enfermagem, nos métodos de desenvolvimento das ações de enfermagem, no conceito, no contexto e nas metas da enfermagem.

$\mathrm{Na}$ complexidade de conceituações, os termos utilizados devem focalizar as propostas da teoria bem como as ações de enfermagem. Analisamos o conceito de Enfermagem e outros interrelacionados definidos na TUDCC, os quais estão no texto-base do trabalho final de mestrado, escolhida como contexto deste estudo.

Leininger (1991, p. 47), define Enfermagem:

Uma profissão e uma disciplina cientifica aprendida e humanista enfocada no fenômeno e nas atividades do cuidado humano para assistir, apoiar, facilitar ou capacitar individuos a grupos a manterem ou readquirir seu bem-estar (ou saúde) em forma culturalmente siguificativas e benéficas ou para ajudar a pessoa a enfrentar a deficiência ou a morte.

Analisando os atributos de clareza e consistência, procuramos entender o significado das partes e, ao final, entender a interrelação dos termos expostos na concretude do conceito de enfermagem e ainda responder aos questionamentos propostos sobre o pensar e o fazer da enfermagem, a partir da definição do conceito. 


\section{CONSTRUTOS DAS DIMENSÖES ANALISADAS}

\section{ANÁLISE DO CONCEITO DE ENFERMAGEM TRANSCULTURAL}

Para analisar conceitos é preciso associar os significados das palavras no contexto referenciado. Assim, o conceito de enfermagem será decomposto e explorado de acordo com o sentido das palavras. Uma profissão - atividade ou ocupação especializada da qual se podem tirar os meios de subsistência (FERREIRA, 1989). Também considerada uma disciplina cientifica - várias indicações caracterizam a enfermagem como disciplina. Melleis (1997) a define assim, pela formação do seu corpo de conhecimento próprio, constituido a partir da prática e de experiências cientificas. Neste sentido, está incluida a enfermagem como uma profissão aprendida pelo desenvolvimento da prática fundamentada em um conhecimento em continua transformação. Enfermagem é considerada por Florence Nightingale, como arte e ciência que requer do profissional, além do conhecimento formal e cientifico, vocação e desenvolvimento do potencial criativo e intuitivo (SILVA, 1995). Toda constituição teórica advém de uma prática, a qual é vivenciada, testada ou experimentada. Assim, evolui a Ciência da enfermagem. Humanista - A enfermagem é humanista no contexto da proposta de entender o homem como ser único, de compreender as pessoas em sua existência, em seu mundo, a fim de ajudá-las em várias situações de saúde-doença, percebendo esses seres de forma integral com seus aspectos culturais (LEININGER, 1991). O sentido humanistico refere-se a qualquer movimento filosófico que fundamenta a natureza humana ou os limites e interesses do homem (ABBAGNANO, 1999). A enfermagem é enfocada no fenômeno, ou seja, nos fatos ligados ao ser humano e sua saúde na perspectiva da profissão/ciência. Esses fenômenos são definidos em várias circunstâncias e ações amplamente aceitas e divulgadas como cuidado humano, constitutivo da essência da enfermagem, sendo definido como "... um fenômeno abstrato e concreto relacionado à assistência, o apoio ou à capacitação de experiências ou de comportamentos para outros ou por outros com necessidades evidentes ou antecipados para melhorar uma condição humana ou forma de vida" (LEINIGER,1991, p. 46).

Leininger (1991) destaca o Cuidado, assegurando ser a essência, vale dizer, o enfoque dominante e central da enfermagem que distingue as ações e atividades da enfermeira. Estas são direcionadas para assistir, apoiar, facilitar e capacitar. $\mathrm{O}$ assistir é entendido como ação que tem vários sentidos no enfoque do cuidado humano. Estar presente, apoiar ver, testemunhar, observar, proteger, acompanhar na qualidade de assistente. (FERREIRA, 1989; FLORENZANO 1999). De acordo com os mesmos autores apoiar tem sentido de dar suporte, no sentido empregado por Leininger também assume uma amplitude de intervenções junto ao cliente, dependendo da sua necessidade consoante às idéias - evidentemente com torneios diferentes - de Ferreira (1989) e Florenzano (1999). Facilitar - significa tornar mais fácil (FERREIRA, 1989). No conceito, relacionando à saúde, é tornar mais fácil ao cliente a compreensão e ação dos atos imprescindiveis para manter ou recuperar a saúde. Capacitar denotativo de tornar o cliente hábil para cuidar de si, conscientizá-lo a ter atitudes e decisões para que mantenha ou readquira a saúde, entendida como bem-estar ligado a própria pessoa.

O significado de facilitar e capacitar propõe ao cliente decisão e participação no cuidado com a sua saúde, dando-lhe oportunidade de agir conforme suas experiências, sendo ajudado pela enfermeira ou equipe de saúde. Estas ações são dinâmicas e atendem ao individuo, ou grupos, os quais estão inseridos em uma familia. A Teoria Transcultural enseja o atendimento a estes grupos numa perspectiva de totalidade. Esse sentido orienta o cuidado ao cliente não apenas na doença, mas estimula as ações preventivas e de promoção da saúde, pois o objetivo da enfermagem é suprir cuidados ao cliente que experimenta a doença ou problemas potenciais que interferem na normalidade da sua higidez. A enfermagem em formas culturalmente significativas e benéficas. Este titulo evidencia os propósitos da TUDCC, ao relatar a diversidade de padrões e valores culturais implicados no cuidado humano, sendo, portanto, 
fundamental à enfermagem capacitar-se para entender esta diversidade de culturas, aprofundando o conhecimento com enfoque ao cuidado cultural.

CONCEITO DE ENFERMAGEM E DE CUIDAR/CUIDADO - INTERRELAÇÃO COM AS FORMULAÇÕES TEÓRICAS ESPOSADAS NO ESCRITO-BASE DO ENSAIO DE MESTRADO

Analisar o conceito de Enfermagem presente na TUDCC implica destacar aspectos do cuidado, pela relação intrínseca entre ambos. A partir do recorte da dissertação de mestrado em estudo, constatamos a interrelação dos achados com a teorização embasada na Teoria Transcultural, de Leininger. A pesquisa em análise envolve vários conceitos de cuidar/cuidado, são definidos na perspectiva do conceito de Enfermagem há pouco analisado. Na intenção de entender as construções teóricas presentes, destacamos algumas que atendem a finalidade do estudo: "O cuidar é uma fonte de sabedoria humana, produzida pela própria necessidade do homem. Envolve a responsabilidade com o outro, como projeto de vida. Para isso é necessário, então que haja encontro, amor e respeito pela pessoa que é cuidada" (QUEIROZ, 1998, p.102). Para a autora: "é uma experiência humana presente em várias situações do continuum vivermorrer e conseqüentemente do processo saúde doença. O cuidado possibilita ação para viver, ser saudável, em que está presente a comunicação, muitas vezes a troca de experiência entre as pessoas, seja baseada em conhecimento cientifico ou popular" (ibid., p.101).

O fenômeno do cuidado é assumido tanto por profissionais como por pessoas que assimilam culturalmente maneiras de cuidar, permeadas pelo diálogo e encontro entre o cuidado e o ser cuidado. É definido no conceito de Enfermagem o valor das experiências desses sujeitos, pois o cuidado é uma ação da enfermagem e da saúde, inspirada em um saber construido socialmente, consubstanciado em diversas culturas. Nesse sentido, lembramos o que é escrito por Leininger (1991): que os sistemas de saúde são diversificados e o cuidado de enfermagem permeia o sistema popular e o sistema profissional. Portanto, para prestar o cuidado cultural, é necessário, além de conhecer e respeitar, às vezes, é preciso agir em consonância com os padrões culturais do cliente, mas sem prejudicá-lo. Dessa forma, age e toma decisões, apropriando-se de conceitos como manter/preservar, acomodar/negociar, repadronizar/ reestruturar o cuidado, tornando-o congruente com a cultura. O significado e justificativa desses conceitos estão presentes na TUDCC, radicada na Antropologia Cultural e Filosófica.

O cuidado cultural é expresso de formas diversas: tecnicamente e/ ou por meio de processos interacionais, comunicativos e educacionais. Assim, apreendemos nos escritos que "É indispensável à enfermeira promover meios e estimular a mãe a se integrar no tratamento e recuperação da criança, orientando adequadamente de acordo com a sua cultura, ensejandoIhe a oportunidade de melhorar a qualidade de saúde de seus membros" (op. cit. p.54). Outras citações evidenciam as formulações teóricas da pesquisa de que ora tratamos em harmonia com a teoria: "Crenças e práticas caminham juntas e a partir de uma crença surgem ações de cuidado, positivas ou não. Estas crenças em si devem ser respeitadas. Contudo, podem ser repadronizadas pela enfermeira" (op. cit. p. 116). Esta ação é reconhecida por Leininger, na TUDCC, como Repadronização do cuidado, uma atitude para satisfazer o cliente e maximizar as ações do cuidado. Uma prática contrária é considerada pela teórica como imposição cultural do profissional, não respeitando os valores culturais do cliente. Outra situação de cuidado cultural é descrita por Queiroz (1998 p. 30). "É essencial conhecer as influências culturais envolvidas no comportamento da mãe para entender determinadas condutas realizadas por ela". A autora ressalta que essa forma de agir contribui para uma participação efetiva da familia no cuidado à criança e constitui uma perspectiva de cuidados culturalmente congruentes.

Entendendo que a cultura compreende um conjunto de significados e valores que caracteriza o modo de vida das pessoas, faz-se necessária uma comunicação adequada para "compreender" as atitudes e comportamentos das pessoas, essencialmente, nas ações de educação em saúde. Estas são afirmações constantes no trabalho focalizado, as quais estão 
coerentes com as proposições do conceito que envolve o fenômeno da enfermagem ao definir propósitos claros de assistir, apoiar, facilitar e capacitar individuos ou grupos em sua condição de vida e saúde.

Algumas construções textuais reforçam a idéia de que o "cuidado é uma necessidade fundamental que envolve várias dimensões" (ibid., p.108). Esta asserção serve para argumentar sobre o conjunto de ações expressas no conceito de Enfermagem Transcultural, que orienta um cuidado humanistico e holístico, pois os principios filosóficos dessa teoria levam em consideração esta perspectiva.

Constatamos que, em várias discussões desse estudo, são ressaltadas as ações de cuidado que explicam a enfermagem atuando no processo saúde-doença, admitindo a possibilidade de atender além das necessidades do corpo, mas, integrando corpo-mente e percebendo a pessoa em seu ambiente sociocultural. Assim, encontramos no conceito de Enfermagem uma pluralidade de ações, mas, todas convergem para o cuidado humano indispensável à sobrevivência, ao crescimento e à saúde em suas variadas dimensões.

\section{CONSIDERAÇÕES FINAIS}

O estudo propiciou uma análise sobre o conceito de Enfermagem Transcultural, de Leininger, atendendo em parte os critérios propostos por Barnum (1998). Entendemos que o mundo do conhecimento é inacabado e esse estudo enseja possibilidades de descobrir mais idéias latentes ali, utilizando outros olhares e vertentes do saber para além do senso comum. Entretanto, nessa análise foi possivel perceber que o conceito de Enfermagem, descrito na TUDCC, veicula uma mensagem clara, revelando idéias concretas do que é a Enfermagem. Os termos apresentam-se consistentes, denotando o sentido dos vocábulos no contexto e a harmonia entre eles, de forma a expressar os objetivos da Enfermagem. Por meio da decomposição, identificamos os componentes, decifrando a multiplicidade de significados que caracterizam o conceito. Assim, apreendemos a natureza das ações de Enfermagem, o que ela faz e como deverá fazer, mostrando ser um conceito prescritivo.

Outro ponto analisado foi a interrelação do conceito de Enfermagem definido por Leininger e a construção teórica presente nos resultados da dissertação utilizada como material empírico. Identificamos em várias descriçōes idéias conceituais de Enfermagem Transcultural, assegurando que houve interrelação do referencial da TUDCC, de Leininger com as construções teóricas da autora. Porém, durante a análise notamos que alguns conceitos não se apresentam bem definidos, mas estão estruturados com base na teoria analisada.

O conjunto de enunciados presente no conceito de Enfermagem, de Leininger, e na dissertação, mostram o foco da enfermagem, a relação intrinseca com o cuidado, o qual deve ser desenvolvido segundo a cultura do cliente. Demonstra, ainda, que a Enfermagem é apoiada num corpo de conhecimentos próprios, os quais, embora derivados de outras disciplinas, tornamse especificos à profissão, pois estão apoiados em pressupostos da Enfermagem que orientam formas diferentes de perceber e cuidar do cliente, valorizando a individualidade, a subjetividade e a sua visão de mundo. De certa forma, o conceito elucida as metas da Enfermagem, denotando ser uma profissão diferente da Medicina e de outros misteres que dedicam o seu trabalho em função da qualidade de vida e saúde do ser humano.

Esta construção permitiu ainda, mergulhar em um conteúdo elaborado por uma das autoras, porém o olhar criterioso da outra pesquisadora garantiu controlar a possibilidade de viés, enfim, facilitou a veracidade dos achados. Assim, o estudo possibilitou a validação dos conceitos estudados na utilização da pesquisa.

ABSTRACT : This is an analytic-bibliographic study which analyzes the concept of nursing in Leininger's Theory and the conceptual development of Nursing presented in the master's degree dissertation chosen for this study. Barnum's model for the analysis and evaluation of theories (Barnum 1998) was 
used, in relation to the clarity and consistency of the concept, together with questionings about Nursing. The group of statements presented in Leininger's transcultural nursing concept and in the dissertation shows what is the focus of nursing and the intrinsic relation to the process of looking after somebody, which must be developed coherently with culture. It also demonstrates that Nursing has a body of specific professional knowledge which guides the care for the client, valuing one's individuality, subjectivity and way of seeing the world. The study made it possible to validate concepts taken from the Theory of Universality and Cultural Diversity of caring and their use in the research according to the analyzed criteria.

KEYWORDS: concept of nursing, culture, care

RESUMO: Estudo bibliográfico-analítico, objetivando analisar o conceito de enfermagem da Teoria de Leininger e o desenvolvimento conceitual de Enfermagem presente na dissertaçäo de mestrado escolhida para o referido estudo. Utilizamos o modelo de análise e avaliação de teorias de Barnum (1998), em relação à clareza e consistência do conceito, associado a questionamentos sobre a Enfermagem. O conjunto de enunciados, presentes no conceito de Enfermagem Transcultural de Leininger e na dissertação, mostra o foco da enfermagem e a relação intrinseca com o cuidado, o qual deve ser desenvolvido coerente com a cultura. Demonstra, ainda, que a Enfermagem tem um corpo de conhecimentos especificos à profissão que orienta o cuidado ao cliente, valorizando a individualidade, a subjetividade e a visão de mundo. Concluimos que o estudo proporcionou validar conceitos abstraidos da Teoria da Universalidade e Diversidade Cultural do cuidado e sua utilização na pesquisa, conforme os critérios analisados.

PALAVRAS CHAVE: conceito de enfermagem, cultura, cuidar/cuidado

\title{
REFERÊNCIAS BIBLIOGRÁFICAS
}

ABBAGNANO, N. Dicionário de Filosofia. 2. ed. São Paulo: Martins Fontes, 1999.

BARNUM, B. J. S. Nursing Theory: análisis, application, evaluation. 5. ed. Philadelphia/ New York: Lippincott, 1998.

FERREIRA, A. B. de H. Dicionário da Lingua Portuguesa. 3. ed. Rio de Janeiro: Nova Fronteira, 1989.

FLORENZANO, É. Dicionário Inglês-Português, Português-Inglês. 47. ed. Rio de Janeiro: Ediouro, 1999.

HICKMAN, J.S. Introdução à Teoria de Enfermagem. In: GEORGE, J.B. et al. Teorias de Enfermagem: os fundamentos à prática de Enfermagem. 4. ed. Porto Alegre:Artmed, 2000.

LEININGER, M. Transcultural nursing: concepts, theories, and practics. New York: Jonh Wiley \& Sons, 1978. 1991. Culture care diversity \& universality: a theory of nursing. New York: National League for Nursing Press,

MELLEIS, A. I. Theoretical nursing: development and progress. 3. ed. Philadelphia/ New York: Lippincott, 1997.

QUEIROZ, M. V. O. Só a mãe conhece o filho: um estudo na etnoenfermagem. Fortaleza, 1998. 136p. Dissertação (Mestrado)-Curso de Mestrado em Enfermagem da Universidade Federal do Ceará.

SILVA, A. L. M. O saber nightingaliano no cuidado: uma abordagem epistemológica. In: WALDOW, V. R., LOPES, M. J. M., MEYER, D. E. Maneiras de cuidar, maneiras de ensinar: a enfermagem entre a escola e a prática profissional. Porto Alegre : Artes Médicas, 1995.

\author{
Recebido em maio de 2001 \\ Aprovado em dezembro de 2001
}

R. Bras. Enferm., Brasilia, v. 54, n.4, p. 630-637, out./dez. 2001 